\title{
Irreversible Work of Separation and Heat-Driven Separation
}

\author{
Anatoliy M. Tsirlin ${ }^{\dagger}$ and Vladimir Kazakov*,: \\ Program System Institute, Russian Academy of Science, set. "Botic”, Perejaslavl-Zalesky, Russia 152020, and \\ School of Finance \& Economics, University of Technology, Sydney, NSW 2007 Australia
}

Received: November 14, 2003; In Final Form: February 21, 2004

\begin{abstract}
Estimates of the minimal necessary work of separation for mechanical separation processes and of the minimal necessary heat consumption for heat-driven separation processes with given productivity are derived in this paper. It is shown that for heat-driven processes the productivity is limited, and this limiting productivity is estimated.
\end{abstract}

\section{Introduction}

The minimal amount of energy needed for separation a mixture with a given composition can be estimated using reversible thermodynamics. These estimates turn out to be very loose and unrealistic. They also do not take into account kinetic factors (laws and coefficients of heat and mass transfer, productivity of the system, etc.). In this paper we derive irreversible estimates of the work of separation that take into account all these factors.

The majority of separation systems are open systems that exchange mass and energy with the environment. If mass and heat transfer coefficients (determined by the size and construction of the apparatus) are finite and if the productivity of the system is finite then the processes in such systems are reversible. The energy flows, the compositions of the mass flows, and the productivity of the system are linked via the balance equations of energy, mass, and entropy. The latter also includes entropy production in the system. Minimal energy used for separation corresponds to minimal entropy production in the system subject to various constraints. This allows us to estimate this minimal energy. ${ }^{1}$

There is a qualitative as well as a quantitative difference between the reversible and irreversible estimates obtained in this paper. For example, the irreversible estimate of the work of separation for poor mixtures (where the concentration of one of the components is close to one) tends to a finite nonzero limit, which depends on the kinetics factors. The reversible work of separation for such mixtures tends to zero. The reversible estimate differs from the amount of energy needed in practice for separation of poor mixtures by a factor of $10^{5} .^{2}$

For heat-driven separation processes the novel results obtained in this paper include the estimate of the minimal heat consumption as a function of kinetic factors and the thermodynamic limit on the productivity of a heat-driven separation.

\section{Thermodynamic Balances of Separation Processes and the Link between Energy Consumption and Entropy Production}

Consider the system, shown in Figure 1, where the flow of mixture with rate $g_{0}$, composition $x_{0}$, temperature $T_{0}$, and

* Corresponding author. E-mail:Vladimir.Kazakov@uts.edu.au.

†ussian Academy of Science. E-mail: tsirlin@sarc.botik.ru.

$\div$ University of Technology, Sydney.

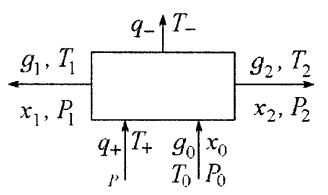

Figure 1. Simplified schematic of thermodynamic balances for separation processes.

pressure $P_{0}$ is separated into two flows with the corresponding parameters $g_{i}, x_{i}, T_{i}, P_{i}(i=1,2)$. The flow of heat $q_{+}$with the temperature $T_{+}$can be supplied, and the flow of heat $q_{-}$with the temperature $T_{-}$can be removed. The mechanical work with the rate (power) $p$ can be supplied.

In centrifuging, membrane separation, and adsorptiondesorption cycles that are driven by pressure variations, no heat is supplied/removed and only mechanical work is spent. In absorption-desorption cycles, distillation, and so forth, no mechanical work is spent, only heat is consumed (heat-driven separation). In some cases the number of input and output flows can be larger. As a rule one can still represent the system as an assembly of separate blocks, whose structure is shown in Figure 1.

2.1. Heat-Driven Separation. Consider a heat-driven separation $(p=0)$ and assume that each of the vectors $\mathbf{x}_{i}=\left(x_{i 1}, \ldots\right.$, $\left.x_{i j}, \ldots, x_{i k}\right), i=0,1,2$, consists of $k$ components which denote the molar fraction of the $j$ th substance in the $i$ th flow. The thermodynamic balance equations of mass, energy, and entropy here take the following form

$$
\begin{gathered}
g_{0} x_{0 j}-g_{1} x_{1 j}-g_{2} x_{2 j}=0, \quad j=1, \ldots, k \\
\sum_{j=1}^{k} x_{i j}=1, \quad i=0,1,2 \\
q_{+}-q_{-}+g_{0} h_{0}-g_{1} h_{1}-g_{2} h_{2}=0
\end{gathered}
$$

where $h_{i}$ is the enthalpy of the $i$ th flow;

$$
\frac{q_{+}}{T_{+}}-\frac{q_{-}}{T_{-}}+g_{0} s_{0}-g_{1} s_{1}-g_{2} s_{2}+\sigma=0
$$

$\sigma$ denotes entropy production. From eq 1 , eq 2 follows that $g_{0}$ $=g_{1}+g_{2}$. After elimination of $g_{0}$ from eqs 3 and 4 and 
introduction of enthalpy increments $\Delta h$ and entropy increment $\Delta s$ we get

$$
\begin{gathered}
q_{+}-q_{-}+g_{1} \Delta h_{01}+g_{2} \Delta h_{02}=0 \\
g_{2} \Delta s_{02}+g_{1} \Delta s_{01}+\frac{q_{+}}{T_{+}}-\frac{q_{-}}{T_{-}}+\sigma=0
\end{gathered}
$$

Here, $\Delta h_{0 i}=h_{0}-h_{i}, \Delta s_{0 i}=s_{0}-s_{i}(i=1,2)$.

Elimination of $q_{-}$using eq 5 and its substitution into eq 6 yields

$$
\sum_{i=1}^{2} g_{i}\left(\Delta s_{0 i}-\frac{\Delta h_{0 i}}{T_{-}}\right)+q_{+}\left(\frac{1}{T_{+}}-\frac{1}{T_{-}}\right)+\sigma=0
$$

and the flow of used heat for heat-driven separation is

$$
q_{+}=\frac{T_{+}}{T_{+}-T_{-}}\left[\sum_{i=1}^{2} g_{i}\left(\Delta s_{0 i} T_{-}-\Delta h_{0 i}\right)+\sigma T_{-}\right]
$$

The first term in the square brackets depends only on the parameters of the input and output flows and represents the reversible work of separation per unit of time (reversible power of separation). The second term there represents the process kinetics and corresponding energy dissipation.

For mixtures that are close to ideal gases and ideal solutions, molar enthalpies and entropies $h_{i}$ and $s_{i}$ in the eqs 3 and 4 can be expressed in terms of compositions and specific enthalpies and entropies of the pure substances. We obtain for each of the flows

$$
\begin{gathered}
\Delta h_{0 i}=\sum_{j=1}^{k}\left[x_{0 j} h_{j}\left(T_{0}, P_{0}\right)-x_{i j} h_{j}\left(T_{i}, P_{i}\right)\right] \\
\Delta s_{0 i}=\sum_{j=1}^{k}\left[x_{0 j} s_{j}^{0}\left(T_{0}, P_{0}\right)-x_{i j} s_{j}^{0}\left(T_{i}, P_{i}\right)-\right. \\
\left.R\left(x_{0 j} \ln x_{0 j}-x_{i j} \ln x_{i j}\right)\right], \quad i=1,2
\end{gathered}
$$

where $R$ is the universal gas constant. The reversible energy consumption here is

$$
\begin{array}{r}
q_{+}^{0}=\frac{1}{\eta_{K}} \sum_{i=1}^{2} g_{i} \sum_{j=1}^{k}\left[\left[x_{0 j} s_{j}^{0}\left(T_{0}, P_{0}\right)-x_{i j} s_{j}^{0}\left(T_{i}, P_{i}\right)-R\left(x_{0 j} \ln x_{0 j}-\right.\right.\right. \\
\left.\left.\left.x_{i j} \ln x_{i j}\right)\right] T_{-}+x_{i j} h_{j}\left(T_{i}, P_{i}\right)-x_{0 j} h_{j}\left(T_{0}, P_{0}\right)\right]
\end{array}
$$

We denote here the Carnot efficiency of the ideal cycle of the heat engine as

$$
\eta_{\mathrm{C}}=\frac{T_{+}-T_{-}}{T_{+}}
$$

Condition 7 can be rewritten as

$$
q_{+}=\frac{1}{\eta_{\mathrm{C}}}\left(p^{0}+\sigma T_{-}\right)
$$

Here, $p^{0}$ is the reversible power of separation that is equal to the reversible flow of heat given by eq 9 multiplied by the Carnot efficiency.

When eq 10 was derived we took into account only the irreversibility $\sigma$ of the separation process (the irreversibility of the heat transfer was not taken into account). In reality heat can be supplied/removed with a finite rate only irreversibly.
Any transformation of heat into work with finite heat transfer coefficients and finite power is irreversible. This leads to a lower efficiency than the Carnot efficiency. The closed form expression for this efficiency was obtained in ref 7. It depends on the power $p$ and on heat transfer coefficients for heat supply and heat removal $\alpha_{+}$and $\alpha_{-}$. For the Newton (linear) law of heat transfer it has the form

$$
\begin{aligned}
\eta_{p}=\max \frac{p}{q_{+}}= & 1-\frac{1}{2 T_{+}}\left(T_{+}+T_{-}-\frac{4 p}{\alpha}-\right. \\
& \left.\sqrt{\left(T_{+}-T_{-}\right)^{2}+\left(\frac{4 p}{\alpha}\right)^{2}-8 \frac{p}{\alpha}\left(T_{+}+T_{-}\right)}\right)
\end{aligned}
$$

where it is assumed that there is constant contact of the working body with the heat reservoirs and

$$
\alpha=\frac{4 \alpha_{+} \alpha_{-}}{\alpha_{+}+\alpha_{-}}
$$

It is easy to show that if $p \rightarrow 0$ then $\eta_{p}$ tends to the Carnot efficiency.

Substitution of $\eta_{p}$ instead of $\eta_{C}$ in eq 10 allows us to derive a tighter estimate for the heat consumption in heat-driven separation processes by finding the minimal possible entropy production $\sigma$ subject to various constraints

$$
q_{+} \geq q_{+}^{\min }=\frac{p^{\min }}{\eta_{p}\left(p^{\min }, \alpha, T_{+}, T_{-}\right)}
$$

where

$$
p^{\min }=p^{0}+\sigma^{\min } T_{-}
$$

Conditions 11-14 single out the area of thermodynamically feasible heat-driven separation systems.

Expressions 9 and 10 and eq 7 can be further specified by assuming the constancy of heat capacities, that the mixture is binary, and so forth.

2.2. Mechanical Separation. Consider a separation system that uses mechanical work with rate $p$. Assume that no heat is supplied/removed $\left(q^{+}=q^{-}=0\right)$ and that input and output flows have the same temperature $T$ and the same pressure. Multiplication of eq 6 by $T$ and subtraction of the result from the energy balance (eq 5), where $\left(q_{+}-q_{-}\right)$is replaced with the supplied power $p$, yields

$$
p=T \sigma+g_{0} \sum_{i=1}^{2} \gamma_{i}\left(T \Delta s_{0 i}-\Delta h_{0 i}\right)
$$

Here $\gamma_{i}=g_{i} / g_{0}$.

After taking into account (eq 9) that the enthalpy increment $\Delta h_{0 i}$ in a mechanical separation is zero, we get

$$
p=g_{0} R T\left[\sum_{i=1}^{2} \gamma_{i} \sum_{j=1}^{k} x_{i j} \ln x_{i j}-\sum_{j=1}^{k} x_{0 j} \ln x_{0 j}\right]+T \sigma=p^{0}+T \sigma
$$

The first term in this expression represents the minimal power for separation that corresponds to the reversible process $(\sigma=$ $0)$. This power $p^{0}$ is equal to the difference between the reversible power for complete separation of the input flow $p_{0}^{0}$ $=-g_{0} R T \sum_{j} x_{0 j} \ln x_{0 j}$ and the combined reversible power of separation of the output flows $p_{1}^{0}$ and $p_{2}^{0}$. 


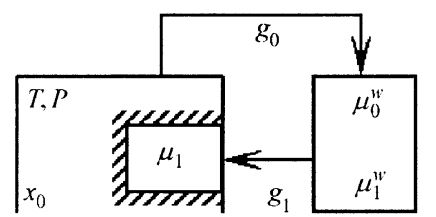

Figure 2. Computational structure of the separation system with reservoir.

Here

$$
p_{i}^{0}\left(x_{i}\right)=-R T g_{0} \gamma_{i} \sum_{j=1}^{k} x_{i j} \ln x_{i j}, \quad i=0,1,2
$$

is the reversible power of separation of the $i$ th flow into pure substances.

\section{Minimal Work of Separation in Irreversible Processes}

3.1. Assumptions and Problem Formulation. Assume that the components of the input mixture are close to ideal gases or ideal solutions. The chemical potential of the $i$ th component can then be written in the following form

$$
\mu_{i}(T, P)=\mu_{0}(T, P)+R T \ln x_{i}, \quad i=1, \ldots, k
$$

where $x_{i}$ is the concentration of the $i$ th component.

First we consider a system that includes three elements (see Figure 2), a reservoir with the time independent temperature $T$, pressure $P$, and vector of concentrations $x_{0}=\left\{x_{01}, \ldots, x_{0 k}\right\}$ (therefore its chemical potential $\mu_{0}$ is also time independent), the finite capacity output subsystem with chemical potential $\mu_{1}$ that depends on the current compositions of the mixture and of the working body that has controllable values of chemical potential $\mu_{0}^{w}$ and $\mu_{1}^{w}$, at the points of contact with reservoir and output subsystem. At the time the intensive variables of the output subsystem coincide with the values of the reservoir's intensive variables, and the number of moles in it is given and equal to $N_{0}$. At time $\tau$ the number of moles $N(\tau)$ and the composition $x(\tau)$ in the output subsystem are given. The mass transfer coefficients between the reservoir and the working body and the working body and the output subsystem are finite and fixed. The minimal necessary work required for the separation is sought.

We do not consider here how to implement the derived optimal dependence of the chemical potential of the working body because of two reasons. First, our main objective is to derive a lower bound on the work of separation. However, imposing constraints on feasible variations of chemical potential would lead to an increase in energy consumption. Second, we will demonstrate that for the majority of mass transfer laws the optimal mass transfer flow is time independent, and its implementation is straightforward.

The work of separation in an isothermal process for an adiabatically insulated system can be found using the Stodola formula in terms of the reversible work $A_{0}$ and the entropy increment $\Delta S$

$$
A=A_{0}+T \Delta S
$$

The reversible work is equal to the increment of the system's internal energy. Since as a result of the process $(N(\tau)-N(0))$ moles of mixture with the composition $x_{0}$ is removed from the reservoir, and the energy of the output subsystem rises because of the increase of the amount of moles in it from $N(0)$ to $N(\tau)$ and its composition from $x_{0}$ to $x_{\tau}$, the total change of the
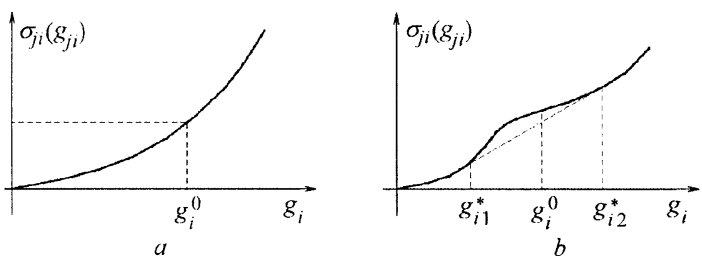

Figure 3. Dependence of the entropy production on the rate for the constant (a) and switching (b) solutions $\left(g_{1 i}^{*}\right.$ and $g_{2 i}^{*}$ are the basic values of the rate).

system's internal energy is

$$
A_{0}=N(\tau) \sum_{i=1}^{k} \Delta \mu_{i}=N(\tau) R T \sum_{i=1}^{k}\left[x_{i}(\tau) \ln x_{i}(\tau)-x_{i 0} \ln x_{i 0}\right]
$$

and it is independent of $N(0)$. Because $A_{0}$ is determined by $N$, $x(\tau), x(0)$, the minimum of $A$ corresponds to the minimum of the entropy increment

$$
\begin{aligned}
\Delta S & =\frac{1}{T} \int_{0}^{\tau} \sum_{i=1}^{k}\left[g_{0 i}\left(\mu_{0 i}-\mu_{i}^{w}\right)+g_{1 i}\left(\mu_{i}^{w}-\mu_{1 i}\right)\right] \mathrm{d} t \\
& =\frac{1}{T} \int_{0}^{\tau} \sum_{i=1}^{k}\left(g_{0 i} \Delta \mu_{0 i}+g_{1 i} \Delta \mu_{1 i}\right) \mathrm{d} t
\end{aligned}
$$

Because the working body's parameters have the same values at the beginning and at the end of a cycle

$$
\begin{aligned}
\int_{0}^{\tau} g_{i 0} \mathrm{~d} t & =\int_{0}^{\tau} g_{i 1} \mathrm{~d} t \\
N(\tau) x_{i}(\tau)-N(0) x_{i}(0) & =\Delta\left(N x_{i}\right), \quad i=1,2, \ldots, k
\end{aligned}
$$

3.2. Optimal Solution. The problem of minimization of $\Delta S$ subject to constraints (eq 22) on $g_{0 i} \geq 0, g_{1 i} \geq 0$ becomes simpler in a common case where the chemical potentials' increments $\Delta \mu_{0 i}, \Delta \mu_{1 i}$ are unique functions of flows $g_{0 i}$ and $g_{1 i}$, correspondingly. If processes are close to equilibrium then this dependence is linear.

Assume

$$
\Delta \mu_{0 i}=\varphi_{0 i}\left(g_{0 i}\right), \quad \Delta \mu_{1 i}=\varphi_{1 i}\left(g_{1 i}\right)
$$

then the problems 21 and 22 can be decomposed into $2 k$ problems

$$
\begin{array}{r}
\Delta S_{j i}=\int_{0}^{\tau} \sigma_{j i}\left(g_{j i}\right) \mathrm{d} t \rightarrow \min / \int_{0}^{\tau} g_{j i} \mathrm{~d} i=\Delta\left(N x_{i}\right) \quad j=0,1, \\
i=1,2, \ldots, k
\end{array}
$$

where $\sigma_{j i}=g_{j i} \varphi_{j i}\left(g_{j i}\right)$ is the function that determines dissipation.

Problems (eq 23) are averaged nonlinear programming problems. Their optimal solutions $g_{j i}^{*}{ }^{3}$ are either constants and equal to

$$
g_{j i}^{*}=g_{1 i}^{*}=\frac{\Delta\left(N x_{i}\right)}{\tau}
$$

or switches between two so-called basic values on the interval $(0, \tau)$, the solution (eq 24) corresponding to the case where the convex envelope of the function $\sigma_{j i}\left(g_{j i}\right)$ is lower than the value of this function at $g_{j i}^{*}$. Characteristic forms of the function $\sigma_{j i}\left(g_{j i}\right)$ for the constant and switching regimes are shown in Figure 3. 
If the function $\sigma_{i j}$ is concave then the optimal rate $g_{j i}$ is always constant. Let us calculate the second derivative of $\sigma$ on $g$ (we omit subscripts for simplicity). If it is positive then the constancy of the rate in the optimal process is guaranteed.

$$
\sigma^{\prime \prime}(g)=2 \varphi^{\prime}(g)+g \varphi^{\prime \prime}(g) \geq 0
$$

The first term in this expression is always positive because the chemical potentials' difference is the driving force of mass transfer and monotonically depends on the flow. For the majority of laws of mass transfer the inequality (eq 25) holds. In particular, it holds if the flow of mass transfer is proportional to the difference of chemical potentials in any positive degree.

Consider mass transfer flow that depends linearly on the chemical potential difference for all $i, j$. Then

$$
g_{j i}=\alpha_{j i} \Delta \mu_{j i} \Longrightarrow \varphi_{j i}=\frac{g_{j i}}{\alpha_{j i}}
$$

It is clear that the conditions (eq 25) hold and the optimal rates of flows obey equalities 24 .

Equalities 24 hold for any nonswitching solution. The minimal increment of the entropy production for such solution is

$$
\Delta S^{\min }=\sum_{i, j} \Delta S_{j i}^{\min }=\tau \sum_{i, j} \sigma_{j i}\left(\frac{\Delta\left(N x_{i}\right)}{\tau}\right)
$$

and the minimal work of separation is

$$
A_{\min }=A_{0}+\tau T \sum_{i, j} \sigma_{j i}\left(\frac{\Delta\left(N x_{i}\right)}{\tau}\right)
$$

The optimal rates are determined by the initial and final states which allows us to specify the estimate (eq 28).

Near equilibrium the flows obey Onsanger's kinetics (eq 26), and from eq 28 it follows that

$$
\begin{gathered}
A_{\min }=A_{0}+\tau \sum_{i=1}^{k} g_{i}^{2}\left(\frac{1}{\alpha_{0 i}}+\frac{1}{\alpha_{1 i}}\right)=A_{0}+\frac{1}{\tau_{i=1}^{k}} \frac{\Delta^{2}\left(N x_{i}\right)}{\overline{\alpha_{i}}} \\
\overline{\alpha_{i}}=\frac{\alpha_{0 i} \alpha_{1 i}}{\alpha_{0 i}+\alpha_{1 i}}
\end{gathered}
$$

is the equivalent mass transfer coefficient on the $i$ th component and the minimal entropy production is

$$
\sigma_{\min }=\frac{1}{T \tau^{2}} \sum_{i=1}^{k} \frac{\Delta^{2}\left(N x_{i}\right)}{\overline{\alpha_{i}}}
$$

The lower bound for the average power of separation is

$$
p_{\min }=\frac{A_{\min }}{\tau}=\frac{A_{0}}{\tau}+\frac{1}{\tau^{2}} \sum_{i=1}^{k} \frac{\Delta^{2}\left(N x_{i}\right)}{\overline{\alpha_{i}}}
$$

$p_{0}=A_{0} / \tau$ is the reversible power of separation.

If

$$
N(0)=0, \quad \Delta\left(N x_{i}\right)=N x_{i}(\tau)
$$

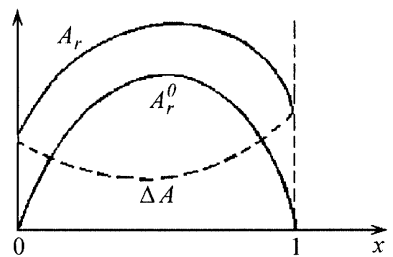

Figure 4. Reversible $\left(A^{0}\right)$ and irreversible $\left(A_{r}\right)$ estimates of the minimal work of separation of binary mixture as functions of key component's concentrations.

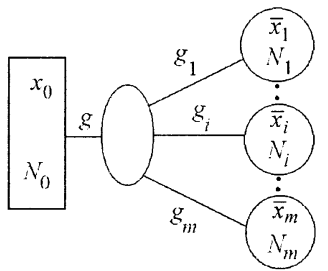

Figure 5. Separation of the system with finite capacity on $m$ subsystems.

then expressions 29 and 32 take the form

$$
\begin{gathered}
A_{\text {min }}=A_{0}+\frac{N^{2}}{\tau} \sum_{i=1}^{k} \frac{x_{i}^{2}(\tau)}{\overline{\alpha_{i}}} \\
p_{\text {min }}=p_{0}+g^{2} \sum_{i=1}^{k} \frac{x_{i}^{2}(\tau)}{\overline{\alpha_{i}}}
\end{gathered}
$$

where

$$
A_{0}=N R T \sum_{i=1}^{k}\left[x_{i}(\tau) \ln x_{i}(\tau)-x_{i} \ln x_{i}\right]
$$

Note that the irreversible estimate of the work of separation (eq 33) does not tend to zero for poor mixtures when the concentration of one of the components tends to one (Figure 4).

If system includes not one but a number of output subsystems then it is clear that the estimate for the minimal work of separation is equal to the sum of the estimates for each subsystem.

$$
A_{\min }=\sum_{j=1} A_{\min }^{j}, \quad p_{\min }=\sum_{j=1} p_{\min }^{j}
$$

The superscript $j$ here denotes the subsystems.

3.3. Separation of a System with Finite Capacity into $m$ Subsystems. Consider a system that is shown in Figure 5. Its initial state is described by the vector of concentrations $x_{0}$, the number of moles of the mixture $N_{0}$, and its final state by the number of moles $\bar{N}_{j}, j=1, \ldots, m$ in each of the subsystems and their concentrations, $x_{j}$. The mass balances yields

$$
\sum_{j=1}^{m} \bar{N}_{j}=N_{0}
$$

$$
\sum_{j=1}^{m} \bar{N}_{j} \bar{x}_{j i}=N_{0} x_{0 i}, \quad i=1,2, \ldots, k
$$


The work in the reversible separation process here is

$$
\begin{aligned}
A_{r}^{0}\left(x_{0}, \bar{x}\right) & =R T\left[\sum_{j=1}^{m} \bar{N}_{j} \sum_{i} \bar{x}_{j i} \ln \bar{x}_{j i}-N_{0} \sum_{i} x_{0 i} \ln x_{0 i}\right] \\
& =A_{r 0}^{0}\left(x_{0}, N_{0}\right)-\sum_{j=1}^{m} A_{r j}^{0}\left(\bar{x}_{j}, \bar{N}_{j}\right)
\end{aligned}
$$

The reversible work of separation is equal to the difference of the reversible work of separation of the initial mixture into pure components and the reversible work of separation for mixtures in each of the subsystems.

We again assume that flows $g_{j}$ have components $g_{j i}$ proportional to the difference of the chemical potential of the subsystem and the working body with the coefficient $\alpha_{j i}$. Here, the condition of minimal work of separation corresponds to the condition of flow constancy

$$
\begin{gathered}
g_{j i}=\frac{\bar{N}_{j} \bar{x}_{j i}}{\tau}, \quad i=1,2, \ldots, k, \quad j=1, \ldots, m \\
\Delta \mu_{j i}=\frac{g_{j i}}{\bar{\alpha}_{j i}}, \quad j=0,1, \ldots, m
\end{gathered}
$$

Here, $\bar{\alpha}_{j i}$ is the equivalent mass transfer coefficient calculated using eq 30 for the flow into the $j$ th output subsystem of the $i$ th component. Similarly as was done above for the system with the reservoir and one finite capacity output subsystem and flows proportional to the final concentrations (eq 39), these concentrations in the output subsystems are time independent and equal to $\bar{x}_{j}$, correspondingly, and the number of moles $\bar{N}_{j}(t)$ depends linearly on time. The power $p$ here is constant

$$
p=\frac{R T}{\tau} \sum_{j=1}^{m} \bar{N}_{j} \sum_{i} \bar{x}_{j i} \ln \frac{\bar{x}_{j i}}{x_{0 i}}+\frac{1}{\tau^{2}} \sum_{j=1}^{m} N_{j}^{2} \sum_{i} \bar{x}_{j i}^{2} / \bar{\alpha}_{j i}
$$

The minimal work of separation for the mixture with concentrations $x_{0}$ into $m$ subsystems with concentrations $\bar{x}_{i}$ over the time $\tau$ is

$$
A_{\mathrm{r}}=R T N_{0} \sum_{j=1}^{m} \gamma_{j} \sum_{i} \bar{x}_{j i} \ln \frac{\bar{x}_{j i}}{x_{0 i}}+\frac{N_{0}^{2}}{\tau} \sum_{j=1}^{m} \gamma_{j}^{2} \sum_{i} \bar{x}_{j i}^{2} / \bar{\alpha}_{j i}
$$

Here, $\gamma_{j}=N_{j} / N_{0}, \bar{\alpha}_{i j}=\alpha_{j i} \alpha_{0 i} /\left(\alpha_{0 i}+\alpha_{j i}\right)$.

The first term here coincides with the reversible work of separation $A_{\mathrm{r}}^{0}$ of the mixture of $N_{0}$ moles with concentration $x_{0}$ into subsystems with number of moles $\bar{N}_{j}$ and concentrations $\bar{x}_{j}$. The second term takes into account irreversibility of the process. $A_{\mathrm{r}}$ decreases monotonically and tends to $A_{\mathrm{r}}^{0}$ when process duration $\tau$ and mass transfer coefficient $\bar{\alpha}_{i j}$ increases.

3.4. Example. Consider separation of the binary mixture into pure components in time $\tau$. In this case $N_{1}=x_{0} N_{0}, N_{2}=$ $\left(1-x_{0}\right) N_{0}$, where $x_{0}$ is the concentration of the key component, $\bar{x}_{11}=\bar{x}_{22}=1$. From the formula 42 we get

$$
\begin{aligned}
& A_{\mathrm{r}}=-R T N_{0}\left(x_{0} \ln x_{0}+\left(1-x_{0}\right) \ln \left(1-x_{0}\right)\right)+ \\
& \frac{N_{0}^{2}}{\tau}\left(\frac{x_{0}^{2}}{\bar{\alpha}_{11}}+\frac{\left(1-x_{0}\right)^{2}}{\bar{\alpha}_{22}}\right)=A_{\mathrm{r}}^{0}\left(x_{0}\right)+\frac{N_{0}^{2}}{\tau}\left(\frac{x_{0}^{2}}{\bar{\alpha}_{11}}+\frac{\left(1-x_{0}\right)^{2}}{\bar{\alpha}_{22}}\right)
\end{aligned}
$$

The estimate (eq 43) was derived in ref 8 by solving the problem of optimal separation of the binary mixture in the given time $\tau$ in Van't Hoff's thought experiment with movable pistons and

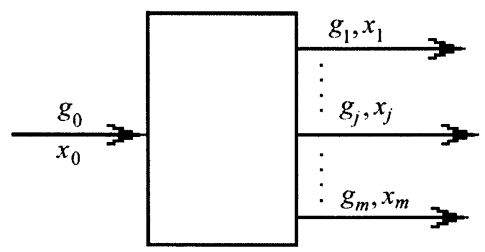

Figure 6. Schematic of a continuous separation system.

semitransparent membrane where $\bar{\alpha}_{11}$ and $\bar{\alpha}_{22}$ are the permeability coefficients on the first and second component. If flows do not depend explicitly on the chemical potentials' differentials, for example, are proportional to the concentrations' differential, then an estimate similar to the one obtained above can be constructed by solving the following auxiliary nonlinear programming problem

$$
\Delta \mu_{i}\left(P_{0}^{i}, P_{\mathrm{i}}\right) \rightarrow \min _{P_{0}, P_{i}} / g_{i}\left(P_{0}^{i}, P_{i}\right)=g_{i}, \quad i=1,2, \ldots
$$

Here, $\left(P_{0}^{i}, P_{i}\right)$ are partial pressures of the components in contacting subsystems that depend on the chemical potentials' differentials $\Delta \mu_{i}$. The flow $g_{\mathrm{i}}$ depends on the same differentials. Minimums in these problems are sought for different values of constant $g_{\mathrm{i}}>0$ and nonpositive $P_{0}^{i}$ and $P_{i}$. We denote the minimal values of the objective in each of these problems $\Delta \mu_{i}^{\min }\left(g_{i}\right)$ as $\Delta \mu_{i}^{*}\left(g_{i}\right)$. This dependence can be used in the estimate (eq 23) of the irreversible work of separation.

3.5. Example. Assume $\Delta \mu=R T \ln \left(P_{0} / P\right), g\left(P_{0}, P\right)=$ $\left(P_{0}-P\right) / \alpha$, and $0<P<P_{\max }$. Let us express $P_{0}$ in terms of $g$ and $P$ :

$$
P_{0 i}=\alpha_{i} g_{i}+P_{i}, \quad i=1,2
$$

$\Delta \mu=R T \ln (\alpha g / P+1)$ attains its minimum at $P=P_{\max } \forall g$. Therefore, $\Delta \mu_{i}^{*}\left(g_{i}\right)=R T \ln \left(\alpha_{i} g_{i} / P_{i \max }+1\right)$.

\section{Potential Application of Obtained Estimates}

We will illustrate the possibilities of the application of the derived estimates.

4.1. Estimate of the Power of Separation in a Continuous Separation System. Consider a continuous separation system with the input flow $g_{0}$ with concentration $x_{0}$ and $m$ output flows $g_{j}(j=1, \ldots, m)$ with concentrations $x_{j}=\left\{x_{j 0}, x_{j 1}, \ldots, x_{j k}\right\}$, (Figure 6 . Here, the temperatures on the input and output flows are close to each other.

Equation 41 allows us to estimate the minimal power required for continuous separation in such system

$$
p_{\min }=\sum_{j=1}^{m} p_{0 j}+g_{0}^{2} \sum_{j=1}^{m} \gamma_{j}^{2} \sum_{i=1}^{k} \frac{x_{j i}^{2}}{\alpha_{j i}}
$$

where

$$
\gamma_{j}=\frac{g_{j}}{g_{0}} \geq 0, \quad \sum_{j=1}^{m} \gamma_{j}=1
$$

$$
p_{0 j}=g_{0} \gamma_{j} R T \sum_{i=1}^{k}\left[x_{j i} \ln x_{j i}-x_{0 i} \ln x_{0 i}\right]=\gamma_{j} M_{j}\left(g_{0}, x_{j}\right)
$$


Mass balance equations yield

$$
\begin{gathered}
\sum_{j=1}^{m} \gamma_{j} x_{j i}=x_{0 i}, \quad i=1, \ldots, k-1, \\
\sum_{i=1}^{k} x_{j i}=1, \quad j=0, \ldots, m
\end{gathered}
$$

The number of conditions (eq 48) is $k-1$, because the concentration of one of the components is determined by the conditions (eq 46).

If the number of flows $m>k$, and their compositions are given, then the removal fractions can be chosen in such a way that the power of separation is minimal subject to constraints (eqs 46 and 48). The Lagrange function of this problem is

$$
L=\sum_{j=1}^{m}\left\{\gamma_{j} M_{j}+\gamma_{j}^{2} r_{j}-\lambda_{0} \gamma_{j}-\sum_{i=1}^{k-1} \lambda_{i} \gamma_{j} x_{j i}\right\}
$$

here

$$
r_{j}\left(g_{0}, x_{j}\right)=g_{0}^{2} \sum_{i=1}^{k} \frac{x_{j i}}{\alpha_{j i}}
$$

$L$ is the concave function on $\gamma_{j}$, and its conditions of stationarity determine the flows that minimize the power for separation for a given flow's compositions

$$
\gamma_{j}^{*}=\frac{\lambda_{0}-M_{j}+\sum_{i=1}^{k-1} \lambda_{i} x_{j i}}{2 r_{j}}, j=1, \ldots, m
$$

We have $k$ linear equations for $\lambda_{0}$ and $\lambda_{i}$

$$
\begin{gathered}
\frac{1}{2}\left[\sum_{j=1}^{m} \frac{\lambda_{0}-M_{j}}{r_{j}}+\sum_{i=1}^{k-1} \lambda_{i} \sum_{j=1}^{m} \frac{x_{j i}}{r_{j}}\right]=1 \\
\frac{1}{2}\left[\sum_{j=1}^{m} x_{j i}\left(\frac{\lambda_{0}-M_{j}}{r_{j}}+\frac{1}{r_{j}} \sum_{i=1}^{k-1} \lambda_{i} x_{j i}\right)\right]=x_{0 i} \quad i=1, \ldots, k-1
\end{gathered}
$$

4.2. Example. Assume $m=3, k=2, g_{0}=1 \mathrm{~mol} / \mathrm{s}, T=$ $300 \mathrm{~K}$, and the compositions and transfer coefficients are

$$
\begin{gathered}
x_{01}=x_{02}=0.5 \\
x_{11}=0.9 ; \quad x_{12}=0.1 ; \quad \bar{\alpha}_{11}=\bar{\alpha}_{12}=0.004 \mathrm{~mol}^{2} /(\mathrm{J} \mathrm{s}) \\
x_{21}=0.3 ; \quad x_{22}=0.7 ; \quad \bar{\alpha}_{21}=\bar{\alpha}_{22}=0.01 \mathrm{~mol}^{2} /(\mathrm{J} \mathrm{s}) \\
x_{31}=0.1 ; \quad x_{32}=0.9 ; \quad \bar{\alpha}_{31}=\bar{\alpha}_{32}=0.06 \mathrm{~mol}^{2} /(\mathrm{J} \mathrm{s})
\end{gathered}
$$

From eq 47 we obtain $M_{1}=910, M_{2}=197, M_{3}=910$, and $r_{1}$ $=205, r_{2}=580, r_{3}=137$.
Equations 51 and 52 for $\lambda$-multipliers take the form

$$
\begin{aligned}
& \frac{1}{2}\left[\frac{\lambda_{0}-M_{1}}{r_{1}}+\frac{\lambda_{0}-M_{2}}{r_{2}}+\frac{\lambda_{0}-M_{3}}{r_{3}}+\right.\left.\lambda_{1}\left(\frac{x_{11}}{r_{1}}+\frac{x_{21}}{r_{2}}+\frac{x_{31}}{r_{3}}\right)\right]=1 \\
& \frac{1}{2}\left[x_{11}\left(\frac{\lambda_{0}-M_{1}}{r_{1}}+\frac{\lambda_{1} x_{11}}{r_{1}}\right)+x_{21}\left(\frac{\lambda_{0}-M_{2}}{r_{2}}+\frac{\lambda_{1} x_{21}}{r_{2}}\right)+\right. \\
&\left.x_{31}\left(\frac{\lambda_{0}-M_{3}}{r_{3}}+\frac{\lambda_{1} x_{31}}{r_{3}}\right)\right]=x_{01}
\end{aligned}
$$

We obtain $\lambda_{0}=894, \lambda_{1}=183$. Their substitution in eq 50 yields $\gamma_{1}^{*}=0.36, \gamma_{2}^{*}=0.64, \gamma_{3}^{*}=0$ and the corresponding estimate for the minimal irreversible power of separation (eq $45)$ is

$$
p_{\min }=718 \mathrm{~J} / \mathrm{S}
$$

4.3. The Selection of the Separation Sequence for a Multicomponent Mixture. In practice, separation of multicomponent mixtures is often realized via a sequence of binary separations. So, a three-component mixture is first separated into two flows, one of which does not contain one of the components. The second flow is then separated into two unicomponent flows. The reversible work of separation (that corresponds to the power $p_{0}$ ) does not depend on the sequence of separation, because $p_{0}$ is determined by the rates and compositions of the input and output flows of the system as a whole. The irreversible component of the power $\Delta p$ in eq 45 depends on the sequence of separation and can be used to find the optimal one.

Consider a three-component mixture with concentration $x_{0}$ $=\left(x_{01}, x_{02}, x_{03}\right)$, and rate $g_{0}$ we set to one. We denote the mass transfer coefficients at the first and second stages of separation as $\alpha_{1}$ and $\alpha_{2}$. They depend on the construction of the apparatus. First, we assume for simplicity that these coefficients do not depend on the mixture's composition (in the general case they do depend on it). We consider irreversible power consumption for two cases:

(a) The first component is first separated, then the second and the third are separated.

(b) The second component is separated, and then the first and the third are separated.

We assume that the separation at each stage is complete. We get up to the constant multiplier

$$
\begin{array}{r}
\Delta p_{a}=\Delta p_{a 1}+\Delta p_{a 2}=x_{01}^{2} / \alpha_{1}+\frac{\left(x_{02}+x_{03}\right)^{2}}{\alpha_{1}}+\left(x_{02}+x_{03}\right)^{2}+ \\
\left(x_{02}^{2} / \alpha_{2}+x_{03}^{2} / \alpha_{3}\right)
\end{array}
$$

The first two terms in this sum represent the loss of irreversibility during the first stage of separation. For $g_{0}=1$ and complete separation the output rates of this stage $g_{1}$ and $g_{2}$ are $x_{01}$ and $\left(x_{02}+x_{03}\right)$, correspondingly.

Consider the first stage of case a for $g_{0}=1$ and complete separation and view the second and third component as the same substance with the output rate $x_{02}+x_{03}=1-x_{01}$. The irreversible expenses (eq 45) are

$$
\Delta p_{a 1}=\frac{x_{01}^{2}}{\alpha_{1}}+\frac{\left(1-x_{01}\right)^{2}}{\alpha_{1}}=\frac{2 x_{01}^{2}+1-2 x_{01}}{\alpha_{1}}
$$


When the second flow is separated into two flows their rates are

$$
g_{22}=\frac{x_{02}}{\left(1-x_{01}\right)}, \quad g_{23}=\frac{x_{03}}{\left(1-x_{01}\right)}
$$

and the irreversible power is

$$
\Delta p_{a 2}=\frac{1}{\alpha_{2}\left(1-x_{01}\right)^{2}}\left(x_{02}^{2}+x_{03}^{2}\right)
$$

The combined irreversible power is

$$
\Delta p_{a}\left(x_{01}, x_{02}\right)=\frac{2 x_{01}^{2}-2 x_{01}+1}{\alpha_{1}}+\frac{x_{02}^{2}+\left(1-x_{01}-x_{02}\right)^{2}}{\alpha_{2}\left(1-x_{01}\right)^{2}}
$$

Similarly in case $\mathrm{b}$ we get

$$
\Delta p_{b}\left(x_{01}, x_{02}\right)=\frac{2 x_{02}^{2}-2 x_{02}+1}{\alpha_{1}}+\frac{x_{01}^{2}+\left(1-x_{01}-x_{02}\right)^{2}}{\alpha_{2}\left(1-x_{02}\right)^{2}}
$$

The differential between these two values is

$$
\begin{aligned}
& \Delta p_{a b}=\Delta p_{a}-\Delta p_{b}=\frac{2}{\alpha_{1}}\left[\left(x_{01}^{2}-x_{02}^{2}\right)-\left(x_{01}-x_{02}\right)\right]+ \\
& \frac{1}{\alpha_{2}\left(1-x_{01}\right)\left(1-x_{02}\right)}\left[\left(1-x_{02}\right)^{2}\left(x_{02}^{2}+x_{03}^{2}\right)-\right. \\
& \left.\left(1-x_{01}\right)^{2}\left(x_{01}^{2}+x_{03}^{3}\right)\right]
\end{aligned}
$$

If $\Delta p_{a b}>0$, then sequence b is preferable.

Note that it is not possible to formulate the general rule to choose the optimal separation sequence for a multicomponent mixture, in particular, on the basis of the reversible work of separation. It is necessary here to compare irreversible losses for each sequence.

4.4. Example. Assume that the composition of the input threecomponent mixture is $x_{01}=0.6, x_{02}=0.3, x_{03}=1-x_{01}-$ $x_{02}$; the mass transfer coefficients are $\alpha_{1}=0.01 \mathrm{~mol}^{2} /(\mathrm{J} \mathrm{s}), \alpha_{2}$ $=0.02 \mathrm{~mol}^{2} /(\mathrm{J} \mathrm{s})$. From eq 55 we find that the difference in power between sequences $\mathrm{a}$ and $\mathrm{b}$ is

$$
\Delta p_{a b}=\Delta p_{a}-\Delta p_{b}=-7.82 \mathrm{~J}
$$

The comparison of the combined minimal irreversible power for the same initial data shows that the power for separation of a mixture using sequence $\mathrm{b}$ is higher than the power used for sequence a, that is, $\Delta p_{a b}<0$.

Thus, sequence a is preferable, and it is better to perform the complete separation by separating the first component.

\section{Limiting Productivity and Minimal Heat Consumption for a Heat-Driven Separation}

In many separation processes a heat engine is used to create the differential of the chemical potential between the working body and the reservoirs (the driving force of mass transfer). Here, the working body is heated during contact with one reservoir and is cooled during contact with the other reservoir. One can represent the heat-driven separation system as a transformer of heat into the work of separation that generates power $p$, consumes heat flow from hot reservoir $g_{+}$, and rejects flow $g_{-}$to the cold reservoir. Heat transfer coefficients for contacts with the hot and cold reservoir $\alpha_{+}$and $\alpha_{-}$are fixed.
It was shown in refs 5 and 6 that the potential of the direct transformation of heat to work is limited and the maximal generated power for the working body with the distributed parameters is

$$
p_{\max }=\bar{\alpha}\left(\sqrt{T}_{+}-\sqrt{T}_{-}\right)^{2}
$$

In this expression $\bar{\alpha}=\left(\alpha_{+} \alpha_{-}\right) /\left(\alpha_{+}+\alpha_{-}\right)$is the equivalent heat transfer coefficient for continuous contact with the reservoirs; $\bar{\alpha}=\left(\alpha_{+} \alpha_{-}\right) /\left({\sqrt{\alpha_{+}}}+\sqrt{\alpha_{-}}\right)^{2}$ is the equivalent heat transfer coefficient for sequential contact.

The maximal power determines the heat flow consumed from the hot reservoir. Further increase of heat consumption for given values of heat transfer coefficients requires an increase of the temperature differential between the reservoirs and the working body and reduces the power.

The dependence of the used power on the productivity of irreversible separation processes is monotonic (eq 45). Therefore, the limiting productivity of heat-driven separation processes corresponds to the maximal possible power produced by transformation of heat into work. Further increase of heat consumption $q_{+}$reduces power and therefore reduces the productivity of separation process.

For the Newton (linear) law of mass transfer and heat-work transformer the dependence of the power on the heat used ${ }^{7}$ is

$$
q^{+}(p)=\frac{p}{\eta_{p}}=\frac{2 p}{\left(\frac{p}{\bar{\alpha} T_{+}}+\eta_{k}\right)+\sqrt{\left(\frac{p}{\bar{\alpha} T_{+}}+\eta_{\mathrm{C}}\right)^{2}-\frac{4 p}{\bar{\alpha} T_{+}}}}
$$

Here, $\eta_{\mathrm{C}}=\left(T_{+}-T_{-}\right) / T_{+}$is the Carnot efficiency, $T_{+}$and $T_{-}$ are the hot and cold reservoir's temperatures, and $\bar{\alpha}=\left(\alpha_{+} \alpha_{-}\right) /$ $\left(\alpha_{+}+\alpha_{-}\right)$is the equivalent heat transfer coefficient.

The minimal heat consumption $q_{+}$as a function of productivity $g_{0}$ for a heat-driven separation can be obtained by substituting expression 57 instead of $p$ in the right-hand side of eq 45. The result holds for $p \leq p_{\max }$ and therefore for $g_{0} \leq g_{0 \max }$. The duration here must not exceed the maximal possible duration.

Substitution of the right-hand side of eq 56 instead of $p$ in eq 45 yields the maximal possible productivity of the system (where $\bar{\alpha}$ is chosen according to the type of contact between the transformer and reservoir). We denote

$$
B=R T \sum_{j} \gamma_{j} \sum_{i} x_{j i} \ln \frac{x_{j i}}{x_{0 i}}, \quad D=T \sum_{j} \gamma_{j}^{2} \sum_{i} \frac{x_{j i}^{2}}{\alpha_{j i}}
$$

We obtain

$$
p_{\max }=\bar{\alpha}\left(\sqrt{T_{+}}-\sqrt{T_{-}}\right)^{2}=B g_{0 \max }+D g_{0 \max }^{2}
$$

and the limiting productivity is

$$
g_{0 \max }=\frac{-B+\sqrt{B^{2}+4 \bar{\alpha} D\left(\sqrt{T_{+}}-\sqrt{T_{-}}\right)^{2}}}{2 D}
$$

Formulas 58 and 59 allow us to estimate the limiting productivity of a heat-driven separation process for Newton's laws of heat transfer between the working body and reservoirs and mass transfer proportional to the differentials in chemical potentials (mass transfer is close to isothermal with the temperature $T$ ).

5.1. Example. Consider heat-driven monoethanamide gas cleansing. One of the components is absorbed by the cold solution from the input gas mixture. This solution is then heated 
and this component is vaporized. The input mixture's parameters are $\bar{T}=350 \mathrm{~K}$, the key component's molar concentration $x=$ 0.5 , the rate of mixture $g_{0}=5 \mathrm{~mol} / \mathrm{s}$. The temperatures of heat supplied/removed are correspondingly $T_{\mathrm{h}}=400 \mathrm{~K}, T_{\mathrm{c}}=300$ $\mathrm{K}$, and the heat transfer coefficients are $\alpha_{+}=8.368 \mathrm{~kJ} /(\mathrm{s} \mathrm{K})$ and $\alpha_{-}=16.736 \mathrm{~kJ} /(\mathrm{s} \mathrm{K})$. The concentrations of the key components in the output flows are $x_{1}=0.9, x_{2}=0.1$; the mass transfer coefficients for each of the components (integral values over the whole contact surface) for the hot and cold reservoir's contacts are $\alpha_{1}=0.07 \mathrm{~mol}^{2} /(\mathrm{kg} \mathrm{s}), \alpha_{2}=0.03 \mathrm{~mol}^{2} /(\mathrm{kg} \mathrm{s})$.

Because the solution circulates and is heated and cooled in turns, the limiting power for transformation of heat into work is given by the expression 56 with the corresponding $\bar{\alpha}$

$$
p_{\max }=20.711 \frac{\mathrm{kJ}}{\mathrm{s}}
$$

The power for separation is given by eq 45 .

We have

$$
p^{0}=R \operatorname{Tg}_{0} \sum_{j=1}^{m} \gamma_{j} \sum_{i} x_{j i} \ln \frac{x_{j i}}{x_{0 i}}=5.397 \frac{\mathrm{kJ}}{\mathrm{s}}
$$

The minimal work required for a system with Onsanger's equations are (see eq 45)

$$
\Delta p=g_{0}^{2} \sum_{j=1}^{m} \gamma_{j}^{2} \sum_{I} \frac{x_{j i}^{2}}{\alpha_{j i}}=7.238 \frac{\mathrm{kJ}}{\mathrm{s}}
$$

Thus, $p=p^{0}+\Delta p=12.636 \mathrm{~kJ} / \mathrm{s}<p_{\max }$. The work needed for separation does not exceed the maximal possible value for given heat transfer coefficients.

Let us estimate the minimal heat consumption. From eq 57 we get

$$
q_{+}=32.426 \frac{\mathrm{kJ}}{\mathrm{s}}
$$

If the temperatures of the input and output flows are not the same then the minimal energy required for separation can be estimated using the thermodynamic balance equations 13 and 14 and the expression for $\sigma^{\mathrm{min}}$ (eq 31 ).

\section{Conclusion}

New irreversible estimates of the in-principle limiting possibilities of separation processes are derived in this paper. They take into account the unavoidable irreversibility caused by the finite rate of flows and heat and mass transfer coefficients. They also allow us to estimate the limiting productivity of a heatdriven separation and to find the most energy efficient separation sequence/regime of separation for a multicomponent mixture.

Acknowledgment. This work is directly supported by a grant from RFFI (Grant 01-01-00020).

\section{References and Notes}

(1) Orlov, V. N.; Rozonoer, L. I. Estimates of Efficiency of Controlled Thermodynamic Processes Based on the Balance Equations of Mass, Energy and Entropy. Abstracts of the 10th All-Union Conference on Control Problems, Moscow, AN SSSR, IPU, 75-76, 1986.

(2) Shambadal, P. Development and Application of the Concept of Entropy; Nauka: Moscow, 1967.

(3) Tsirlin, A. M. Optimal Processes in Thermodynamics and Microeconomics; Nauka: Moscow, 2003.

(4) Tsirlin, A. M.; Mironova, V. A.; Amelkin, S. A.; Kazakov, V. A. Finite-Time Thermodynamics: Conditions of Minimal Dissipation for Thermodynamic Process with Given Rate. Phys. Rev. E 1998, 580, 1.

(5) Novikov, I. I. The Efficiency of Atomic Power Stations. J. Nucl. Energy II (USSR) 1958, 7, 125-128.

(6) Curzon, F. L.; Ahlborn, B. Efficiency of a Carnot Engine at Maximum Power Output. Am. J. Phys. 1975, 43, 22-24.

(7) Rozonoer, L. I.; Tsirlin, A. M. Optimal Control of Thermodynamic Processes. Autom. Remote Control 1983, 70-79, 88-101, 49-64.

(8) Amelkin, S. A.; Butcer, I. M.; Hoffman, K. H.; Tsirlin, A. M. Estimates of Limiting Possibilities of Separation Processes. Theor. Found. Chem. Tech. 1983, 35, 3, 68-75.

(9) Bosnajakovic, F. Technical Thermodynamics; Holt, Rinehart and Winston: New York, 1965; Vol. 2.

(10) Berry, R. S.; Kazakov, V.; Sieniutycz, S.; Szwast, Z.; Tsirlin, A. M. Thermodynamic Optimization of Finite Time Processes, Wiley: Chichester, U.K., 1999. 